\title{
Surgery or radiotherapy for stage I lung cancer? An intention-to-treat analysis
}

\author{
Katie L. Spencer (10) ${ }^{1,2,3}$, Martyn P.T. Kennedy ${ }^{1,3}$, Katie L. Lummis ${ }^{1}$, \\ Deborah A.B. Ellames ${ }^{1}$, Michael Snee ${ }^{1}$, Alessandro Brunelli ${ }^{1}$, \\ Kevin Franks ${ }^{1}$ and Matthew E.J. Callister ${ }^{1}$ \\ Affiliations: \\ ${ }^{1}$ Leeds Teaching Hospitals NHS Trust, Leeds, UK. \\ ${ }^{2}$ Cancer Epidemiology Group, Leeds Institute of Cancer and Pathology, University of Leeds, Leeds, UK. \\ ${ }^{3}$ These two authors are joint first authors. \\ Correspondence: \\ Katie L. Spencer, Cancer Epidemiology Group, Faculty of Medicine and Health, Level 11, Worsley Building, \\ University of Leeds, Clarendon Way, Leeds, LS2 9JT, UK. E-mail: katie.spencer1anhs.net
}

@ERSpublications

No difference seen in cancer- and treatment-specific survival following surgery and SABR. Inferior overall survival may reflect unobserved confounding. Further prospective work should assess quality of life outcomes to support shared decision making. http://ow.ly/7s5t30njP0B

Cite this article as: Spencer KL, Kennedy MPT, Lummis KL, et al. Surgery or radiotherapy for stage I lung cancer? An intention-to-treat analysis. Eur Respir J 2019; 53: 1801568 [https://doi.org/10.1183/ 13993003.01568-2018].

\section{ABSTRACT}

Introduction: Surgery is the standard of care for early-stage lung cancer, with stereotactic ablative body radiotherapy (SABR) a lower morbidity alternative for patients with limited physiological reserve. Comparisons of outcomes between these treatment options are limited by competing comorbidities and differences in pre-treatment pathological information. This study aims to address these issues by assessing both overall and cancer-specific survival for presumed stage I lung cancer on an intention-to-treat basis. Methods: This retrospective intention-to-treat analysis identified all patients treated for presumed stage I lung cancer within a single large UK centre. Overall survival, cancer-specific survival, and combined cancer and treatment-related survival were assessed with adjustment for confounding variables using Cox proportional hazards and Fine-Gray competing risks analyses.

Results: 468 patients (including 316 surgery and 99 SABR) were included in the study population. Compared with surgery, SABR was associated with inferior overall survival on multivariable Cox modelling (SABR HR 1.84 (95\% CI 1.32-2.57)), but there was no difference in cancer-specific survival (SABR HR 1.47 (95\% CI 0.80-2.69)) or combined cancer and treatment-related survival (SABR HR 1.27 (95\% CI 0.74-2.17)). Combined cancer and treatment-related death was no different between SABR and surgery on Fine-Gray competing risks multivariable modelling (subdistribution hazard 1.03 (95\% CI 0.59$1.81)$ ). Non-cancer-related death was significantly higher in SABR than surgery (subdistribution hazard 2.16 (95\% CI 1.41-3.32)).

Conclusion: In this analysis, no difference in cancer-specific survival was observed between SABR and surgery. Further work is needed to define predictors of outcome and help inform treatment decisions. 


\section{Introduction}

Lung cancer has the third highest cancer incidence in the UK and the highest mortality [1]. For medically operable patients with stage I disease, surgical resection with mediastinal lymph node sampling is the standard of care. Overall survival is $54-73 \%$ at 5 years [2-6], with 5-6\% locoregional recurrence [5-7] and $17-18 \%$ overall recurrence $[5,6]$. Lung cancer is, however, associated with advanced age and comorbidity [8]. This results in higher perioperative mortality risk [9], often precluding surgery [8]. Sublobar resection can be considered for patients at higher risk of surgical morbidity or mortality [10].

Radiotherapy is an alternative treatment for medically inoperable patients or those declining surgery. Historical series of conventionally fractionated radiotherapy (CFRT) have shown poorer outcomes than surgical resection [11-13]. Stereotactic ablative body radiotherapy (SABR), however, uses much higher biologically equivalent doses than CFRT, providing improved overall survival with lower morbidity in eligible patients $[14,15]$. Additionally, a large population-based study in the Netherlands demonstrated an increase in rates of radical treatment, stable surgical resection rates and improved overall survival for older patients with early-stage nonsmall cell lung cancer (NSCLC) when SABR was introduced [16]. In England, SABR is only commissioned for peripherally located lesions. While more centrally located early-stage lung cancers can be treated with SABR, there is considerable debate on the optimal dose and the safety of this approach, particularly for "ultra-central" lesions. The full results of the North America NRG Oncology/ Radiation Therapy Oncology Group 0813 and the Scandinavian HILUS trials are awaited and may provide further information $[17,18]$.

Surgical patients tend to be younger with less comorbidity than those undergoing SABR or CFRT $[3,19]$. As such, comparisons between reported outcomes of cohort studies are limited by selection bias and the confounding effect of unobserved characteristics. Additionally, information bias may occur; definitive pathological staging is available for a majority of surgical patients, with upstaged patients usually being excluded from comparisons, while occult nodal disease may go undetected in the SABR population despite positron emission tomography-computed tomography (PET-CT) and endobronchial ultrasound nodal aspirate-based staging. Conversely, there is a risk that unbiopsied benign disease in SABR cohorts may result in better than expected cancer-specific survival. As a consequence, retrospective comparisons are limited by inevitable case selection differences and staging uncertainty. The extent to which survival differences reflect treatment efficacy has not been definitively demonstrated. Meta-analyses of cohort studies comparing surgery with SABR have found comparable mortality and local control rates when adjusted for medical operability, age and comorbidity [3, 19]. Conversely, a recent propensity-matched study, using SEER (Surveillance, Epidemiology and End Results) data, has suggested superior outcomes with surgery compared with SABR for patients with stage IB disease [20].

Three randomised controlled trials comparing surgery and SABR in higher risk medically operable patients have, unfortunately, failed to recruit (ROSEL (ClinicalTrials.gov identifier NCT00687986), STARS (ClinicalTrials.gov identifier NCT00840749) and ASOSOG-RTOG (ClinicalTrials.gov identifier NCT01336894)) and the results of the feasibility SABRTooth trial (ClinicalTrials.gov identifier NCT02629458) are awaited. An intention-to-treat analysis of pooled data from ROSEL and STARS demonstrated superior 3-year overall survival with SABR, although small sample size and short follow-up mean these results should be interpreted with caution [21]. Further randomised studies (including STABLE-MATES (ClinicalTrials.gov identifier NCT02468024), POSTIlV (ClinicalTrials.gov identifier NCT01753414) and VALOR (ClinicalTrials. gov identifier NCT0298476)) are ongoing.

Given the clear challenges faced in randomising patients in this situation [22], this study aims to analyse the outcomes following radical treatment of presumed stage I lung cancer in order to assess cancer-specific mortality and treatment effect within an intention-to-treat population.

\section{Materials and methods}

This single-centre retrospective cohort study included all consecutive patients diagnosed and treated with curative intent for presumed stage I lung cancer at Leeds Teaching Hospitals NHS Trust (LTHT; Leeds, UK) between January 2008 and May 2013. All patients who received radical lung cancer treatment were identified using an automated search of the local electronic health record. Pre-treatment stage was confirmed using radiology and multidisciplinary team (MDT) records using the 7 th edition of the American Joint Committee on Cancer tumour/node/metastasis staging for lung cancer (cancerstaging.org). Manual review of records identified additional patients treated surgically for presumed stage I lung cancer, but subsequently diagnosed with benign pathology. All patients underwent PET-CT staging. All patients with presumed stage I disease pre-treatment were included in the study cohort. Patients without histological confirmation needed to a have 1) a PET-positive lesion and/or serial growth on imaging; 2) a contraindication to, or a failed, biopsy; and 3) MDT consensus supporting a lung cancer diagnosis before having radiotherapy. Demographic and baseline clinical data (including performance status) were 
collected. Comorbidity and physiological reserve, however, were not systematically recorded, and therefore were not considered within this analysis.

LTHT is a large publically funded UK teaching hospital. It has a local catchment population of 780000 , and is the regional referral centre for a further 2.7 million for both surgery and radiotherapy. All patients are managed by a lung cancer-specific MDT in line with national guidelines. Prior to May 2009, CFRT was the standard nonsurgical treatment for stage I lung cancer in LTHT. At this time, SABR was implemented as the standard of care for patients with medically inoperable peripheral stage I disease [23, 24]. CFRT remained the standard of care for more central lesions.

Standard follow-up after radical treatment of lung cancer during this period included clinic review with chest radiograph 3-monthly in year 1, 6-monthly in year 2 and annually in years 3-5 [25]. Recurrence was defined on radiological or clinical grounds and either pathologically confirmed or clinically accepted by lung cancer MDT review. New primary lung cancers, diagnosed on the basis of histological subtype, immunohistochemistry or clinically, were not considered recurrences $[26,27]$. The site of recurrence was recorded as local, nodal or metastatic. Local recurrence included recurrence at the resection margin following surgical resection, or within the lung parenchyma of the same lobe following sublobar resection or radiotherapy (i.e. potentially $>2-3 \mathrm{~cm}$ from the gross tumour volume). Nodal recurrence included all intrathoracic lymph nodes. Extrathoracic lymph nodes, different ipsilateral lobe, contralateral lung, pleural effusions or distant organ recurrence were considered metastatic. Date and cause of death were recorded. The latter was determined through retrospective case note review by two independent investigating doctors and defined as treatment-related, cancer-related (death following diagnosis of recurrent cancer) or due to comorbidity (death without recurrent cancer). No interobserver disagreement occurred.

\section{Statistical analysis}

All analyses were based on delivered treatment modality (surgery, SABR or CFRT). Baseline characteristics of the treatment groups were compared using the Chi-squared test and two-sided t-test for age. Overall survival, cancer-specific survival, and combined cancer and treatment-related survival were determined, and Kaplan-Meier survival curves produced. Censoring was at the point of data collection for overall survival; at data collection, death due to comorbidity or treatment-related death for cancer-specific survival; and at data

\section{Identified through search of electronic health records}

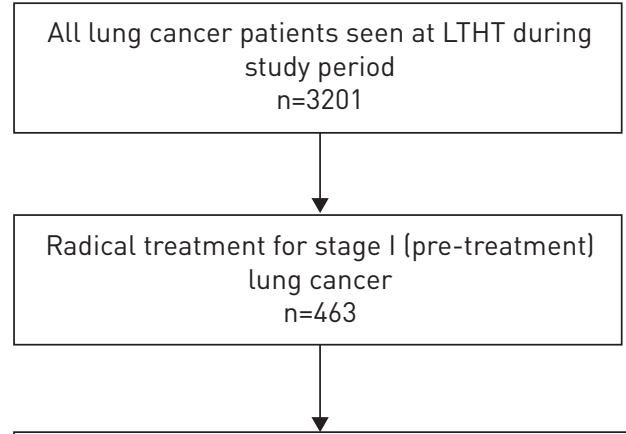

Identified through manual case note review of patients undergoing thoracic surgery following lung MDT review

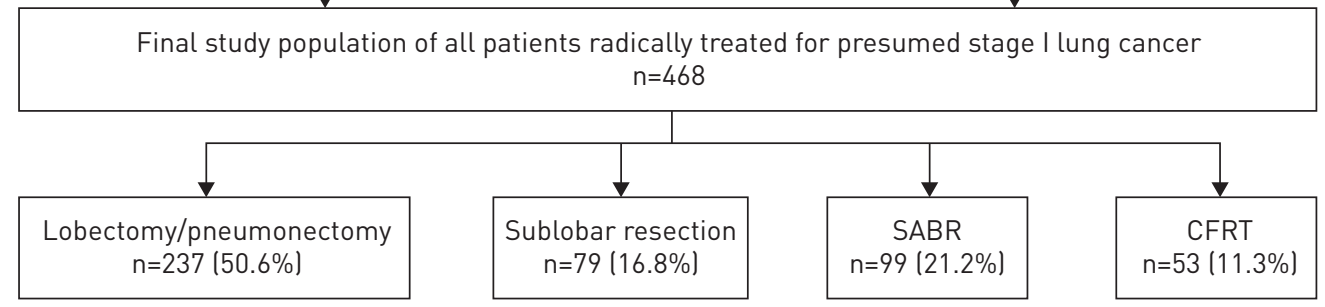

Benign resection for what was thought to be stage I lung cancer $\mathrm{n}=5$

Post-operative pathology:

$\mathrm{n}=247(78.1 \%)$ confirmed stage I

$\mathrm{n}=64(20.3 \%)$ higher stage

$\mathrm{n}=5(1.6 \%)$ benign

FIGURE 1 CONSORT diagram illustrating the study cohort. MDT: multidisciplinary team; LTHT: Leeds Teaching Hospitals NHS Trust; SABR: stereotactic ablative body radiotherapy; CFRT: conventionally fractionated radiotherapy. 
collection or death due to comorbidity for combined cancer and treatment-related survival. Cancer-specific survival therefore encompasses deaths following cancer recurrence only, while combined cancer and treatment-related survival encompasses deaths due to either cancer treatment or recurrence [28]. The factors associated with these outcomes were considered using univariable and multivariable Cox proportional hazards models. Histological subtype was excluded from multivariable models due to collinearity of the unknown subgroup with the radiotherapy treatment arms. Heterogeneity of treatment effect on combined cancer and treatment-related survival with age and performance status was assessed using interaction terms and by examining separate treatment cohorts

To address possible concerns about the suitability of Cox modelling in a situation where censoring differs widely between cohorts, the multivariable analysis was replicated using a Fine-Gray competing risks model [29]. The cumulative incidence function of death due to varying causes was plotted.

Analyses were repeated excluding patients without histological confirmation prior to treatment.

All statistical analyses were carried out in Stata IC version 14 (StataCorp, College Station, TX, USA).

\section{Ethical approval}

This study was approved as a local evaluation of treatment outcomes by the LTHT Research and Innovation department.

\begin{tabular}{|c|c|c|c|c|}
\hline & Surgery & SABR & CFRT & p-value \\
\hline Subjects & 316 & 99 & 53 & \\
\hline Age years & 69.9 (57.12-82.7) & $76.7(63.5-90.0)$ & $76.5(63.8-89.3)$ & \\
\hline \multicolumn{5}{|l|}{ Age group years } \\
\hline $30-59$ & $47(14.87)$ & $4(4.04)$ & $0(0.00)$ & \multirow[t]{4}{*}{$<0.001$} \\
\hline $60-69$ & $112(35.44)$ & $21(21.21)$ & $12(22.64)$ & \\
\hline $70-79$ & $127(40.19)$ & $42(42.42)$ & $20(37.74)$ & \\
\hline$\geqslant 80$ & $30(9.49)$ & 32 (32.32) & $21(39.62)$ & \\
\hline Female & $176(55.70)$ & $44(44.44)$ & $30(56.60)$ & 0.050 \\
\hline \multicolumn{5}{|l|}{ Histology } \\
\hline Squamous cell & $95(30.06)$ & $21(21.21)$ & 16 (30.19) & \multirow[t]{4}{*}{$<0.001$} \\
\hline Other & $215(68.04)$ & 35 (35.35) & 17 (32.08) & \\
\hline Benign & $5(1.58)$ & $0(0.00)$ & $0(0.00)$ & \\
\hline Unknown & $1(0.32)$ & 43 (43.43) & 20 (37.74) & \\
\hline \multicolumn{5}{|c|}{ Pre-operative stage } \\
\hline IA & $197(62.34)$ & 68 (68.69) & $23(43.40)$ & \multirow[t]{10}{*}{0.252} \\
\hline \multicolumn{4}{|c|}{ Post-operative stage } & \\
\hline IA & $140(44.30)$ & & & \\
\hline IB & $107(33.86)$ & & & \\
\hline$\| A$ & $36(11.39)$ & & & \\
\hline IIB & $17(5.38)$ & & & \\
\hline IIIA & $9(2.85)$ & & & \\
\hline IIIB & $1(0.32)$ & & & \\
\hline IV & $1(0.32)$ & & & \\
\hline Benign & $5(1.58)$ & & & \\
\hline \multicolumn{5}{|c|}{ WHO performance status } \\
\hline $0-1$ & 263 (83.23) & $41(41.41)$ & 17 (32.08) & \multirow[t]{3}{*}{$<0.001$} \\
\hline 2 & $45(14.24)$ & 29 (29.29) & 20 (37.74) & \\
\hline $3-4$ & $8(2.53)$ & 29 (29.29) & $16(30.19)$ & \\
\hline \multicolumn{5}{|l|}{ Year } \\
\hline 2008 & 55 (17.41) & $0(0.00)$ & $9(16.98)$ & \multirow[t]{6}{*}{$<0.001$} \\
\hline 2009 & $51(16.14)$ & 8 (8.08) & $6(11.32)$ & \\
\hline 2010 & $46(14.56)$ & 19 (19.19) & 13 (24.53) & \\
\hline 2011 & 54 (17.09) & 30 (30.30) & $7(13.21)$ & \\
\hline 2012 & 72 (22.78) & 28 (28.28) & $14(26.42)$ & \\
\hline 2013 & 38 (12.03) & 14 (14.14) & 4 (7.55) & \\
\hline
\end{tabular}

Data are presented as $n$, median (interquartile range) or $n(\%)$, unless otherwise stated. SABR: stereotactic ablative body radiotherapy; CFRT: conventionally fractionated radiotherapy; WHO: World Health Organization. Histological subtype was not significantly different between the cohorts when those without histological confirmation were excluded ( $p=0.379)$. $p$-values represent the comparison between surgery and SABR. 


\section{Results}

3201 patients diagnosed with lung cancer at LTHT commenced treatment between January 2008 and May 2013. Of these, 463 were treated with curative intent for presumed stage I lung cancer (pre-treatment stage). A further five patients underwent surgery for presumed stage I NSCLC with subsequent benign histology. The final study population consisted of 468 patients. 316 individuals underwent surgical resection (67.4\%), 99 (21.2\%) SABR and 53 (11.3\%) CFRT (figure 1). Of the surgically resected patients, $268(84.8 \%)$ patients underwent lobectomy or pneumonectomy and 48 (15.2\%) patients underwent sublobar resection (of which seven (14.6\%) patients underwent anatomical segmentectomy). Median follow-up was 4.9, 3.8 and 4.7 years following surgery, SABR and CFRT, respectively.

Compared with the SABR cohort, the surgically treated patients were younger $(p<0.001)$ with better World Health Organization performance status $(\mathrm{p}<0.001)$ (table 1). Pre-treatment confirmation of malignancy was available in $56(56.6 \%)$ patients treated with SABR and $34(62.3 \%)$ patients treated with CFRT. Six patients included within the surgical cohort were found to have small cell lung cancer at histological review.

Median overall survival for all radically treated patients was 4.7 years (95\% CI 4.0-5.6). Figure 2a shows overall survival by treatment cohort. The 2-year overall survival was 79.8\% (95\% CI 74.9-83.8), 58.6\% (95\% CI 48.3-67.6) and 54.7\% (95\% CI 40.5-66.9) for the surgery, SABR and CFRT cohorts, respectively. Compared with surgery, SABR and CFRT were associated with significantly worse overall survival on both univariable and multivariable analyses (multivariable HR 1.840 (95\% CI 1.317-2.570) for SABR versus 2.278 (95\% CI 1.539-3.372) for CFRT). In addition, increasing age, male sex, poor performance status, higher stage and unknown histology were all associated with worse overall survival in univariable analysis. Sex and age retained this significance on multivariable analysis (table 2). Excluding patients who did not have a confirmed malignancy prior to treatment resulted in a HR of 1.696 (95\% CI 1.130-2.545) for SABR compared with surgery. A similar but lesser effect was seen in CFRT (HR 2.232 (95\% CI 1.385-3.598)) (supplementary table S4). Compared with surgery, CFRT was associated with significantly worse cancer-specific survival on both univariable and multivariable analyses (multivariable HR 2.281 (95\% CI 1.204-4.319)). Cancer-specific survival following SABR was, however, not significantly different from surgery on either univariable or multivariable analysis (multivariable HR 1.469 (95\% CI 0.802 2.688)). In addition, increasing age and higher stage were associated with worse cancer-specific survival in univariable analyses, but only higher stage retained this on multivariable analysis (supplementary tables S1 and S5).

There were no treatment-related deaths in the SABR cohort; $10(3.2 \%)$ deaths were seen in the surgical cohort and one $(1.9 \%)$ death was seen following CFRT $(\mathrm{p}=0.073)$. Median time to post-surgical death was 20 days, with only one individual dying $>90$ days after surgery. CFRT was associated with a significantly worse combined cancer and treatment-related survival than surgery on both univariable and multivariable analyses (multivariable HR 2.133 (95\% CI 1.208-3.767)), while combined cancer and treatment-related

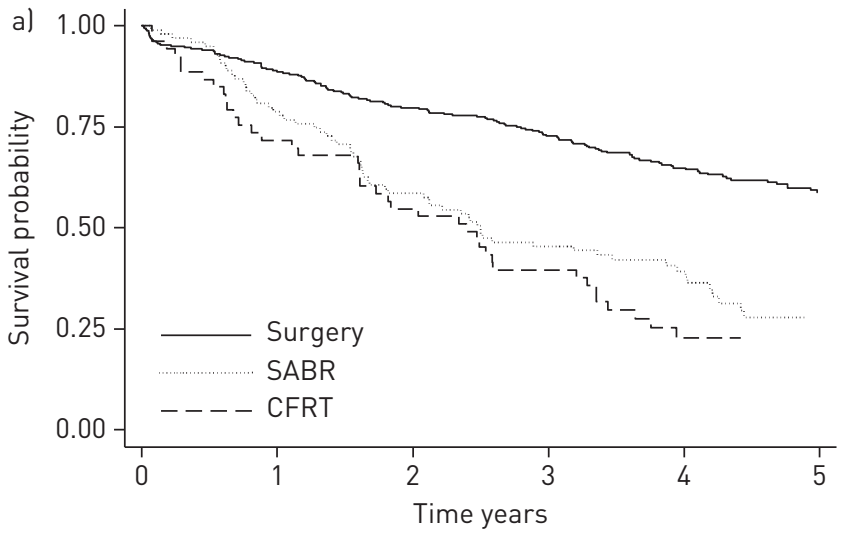

At risk $\mathrm{n}$

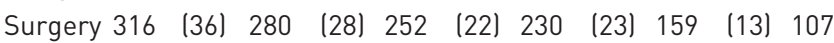

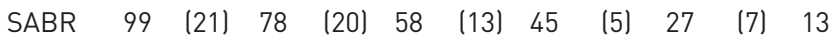

CFRT $\begin{array}{lll}99 & (21) & 78 \\ 53 & (15) & 38\end{array}$

(9) 29
(8) 21

(8) 9

(0) 7

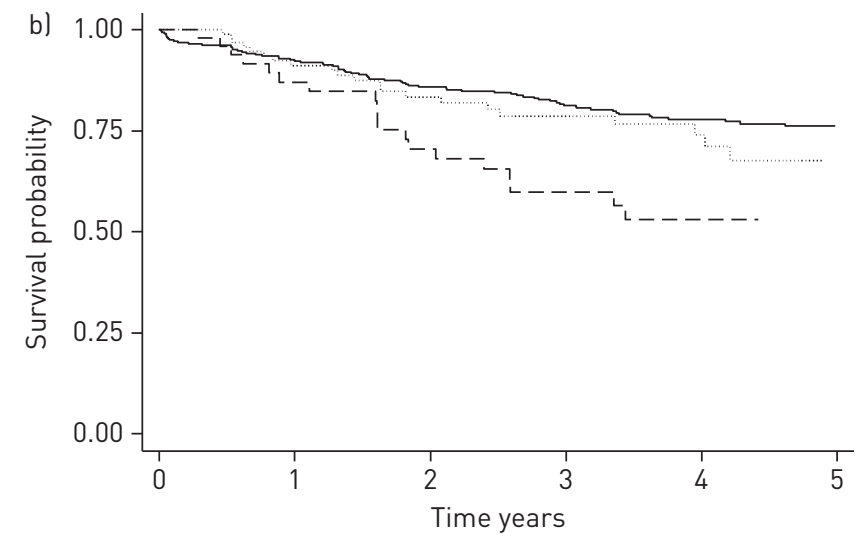

At risk $\mathrm{n}$

$\begin{array}{lccccccccccc}\text { Surgery } & 316 & (24) & 280 & (19) & 252 & (13) & 230 & (9) & 159 & (3) & 107 \\ \text { SABR } & 99 & (8) & 78 & (6) & 58 & (3) & 45 & (2) & 27 & (2) & 13 \\ \text { CFRT } & 53 & (6) & 38 & (7) & 29 & (4) & 21 & (2) & 9 & (0) & 7\end{array}$

FIGURE 2 Kaplan-Meier survival curves showing a) overall survival and b) cancer-specific survival by treatment modality. SABR: stereotactic ablative body radiotherapy; CFRT: conventionally fractionated radiotherapy. Median survival was $2.50(95 \% \mathrm{Cl} 1.80-3.95)$ years in the SABR cohort, 2.40 ( $95 \% \mathrm{Cl}$ 1.60-3.29) years in the CFRT cohort and 6.65 (95\% Cl 5.44-not reached) years in the surgically treated cohort. Number of deaths indicated in brackets. 
TABLE 2 Univariable and multivariable Cox proportional hazards for overall survival

\begin{tabular}{|c|c|c|c|c|}
\hline & \multicolumn{2}{|c|}{ Univariable } & \multicolumn{2}{|c|}{ Multivariable } \\
\hline & HR $(95 \%$ Cl) & $\mathrm{p}$-value & HR $(95 \%$ CI) & p-value \\
\hline \multicolumn{5}{|c|}{ Age group years } \\
\hline$<60$ & 1.000 & & 1.000 & \\
\hline $60-69$ & 1.559 (0.903-2.692) & 0.111 & $1.369(0.790-2.372)$ & 0.264 \\
\hline $70-79$ & $2.317(1.372-3.913)$ & 0.002 & $1.954(1.150-3.319)$ & 0.013 \\
\hline$\geqslant 80$ & $3.063(1.758-5.336)$ & $<0.001$ & $1.897(1.064-3.380)$ & 0.030 \\
\hline \multicolumn{5}{|l|}{ Sex } \\
\hline Female & 1.000 & & 1.000 & \\
\hline Male & $1.614(1.260-2.068)$ & $<0.001$ & $1.670(1.296-2.152)$ & $<0.001$ \\
\hline \multicolumn{5}{|l|}{ Histology } \\
\hline Squamous & 1.000 & & & \\
\hline Other & $0.730(0.548-0.971)$ & 0.031 & & \\
\hline Benign & $0.555(0.136-2.263)$ & 0.412 & & \\
\hline Unknown & $1.951(1.365-2.788)$ & $<0.001$ & & \\
\hline \multicolumn{5}{|c|}{ Pre-operative stage } \\
\hline IA & 1.000 & & 1.000 & \\
\hline IB & $1.392(1.084-1.786)$ & 0.009 & $1.276(0.987-1.650)$ & 0.063 \\
\hline \multicolumn{5}{|c|}{ WHO performance status } \\
\hline $0-1$ & 1.000 & & 1.000 & \\
\hline 2 & 1.697 (1.261-2.283) & $<0.001$ & $1.306(0.952-1.793)$ & 0.098 \\
\hline $3-4$ & 2.244 (1.569-3.209) & $<0.001$ & 1.328 (0.885-1.992) & 0.171 \\
\hline \multicolumn{5}{|l|}{ Treatment } \\
\hline Surgery & 1.000 & & 1.000 & \\
\hline SABR & $2.389(1.787-3.192)$ & $<0.001$ & $1.840(1.317-2.570)$ & $<0.001$ \\
\hline CFRT & 2.831 (1.999-4.008) & $<0.001$ & 2.278 (1.539-3.372) & $<0.001$ \\
\hline
\end{tabular}

WHO: World Health Organization; SABR: stereotactic ablative body radiotherapy; CFRT: conventionally fractionated radiotherapy.

\begin{tabular}{|c|c|c|c|c|}
\hline & \multicolumn{2}{|c|}{ Univariable } & \multicolumn{2}{|c|}{ Multivariable } \\
\hline & HR $(95 \%$ Cl) & p-value & HR $(95 \%$ CI) & p-value \\
\hline \multicolumn{5}{|c|}{ Age group years } \\
\hline$<60$ & 1.000 & & 1.000 & \\
\hline $60-69$ & $1.826(0.810-4.120)$ & 0.147 & $1.670(0.738-3.783)$ & 0.219 \\
\hline $70-79$ & $2.279(1.034-5.024)$ & 0.041 & $1.993(0.898-4.428)$ & 0.090 \\
\hline$\geqslant 80$ & $2.332(0.990-5.493)$ & 0.053 & $1.645(0.674-4.013)$ & 0.274 \\
\hline \multicolumn{5}{|l|}{ Sex } \\
\hline Female & 1.000 & & 1.000 & \\
\hline Male & $1.387(0.959-2.007)$ & 0.082 & $1.386(0.950-2.022)$ & 0.090 \\
\hline \multicolumn{5}{|l|}{ Histology } \\
\hline Squamous & 1.000 & & & \\
\hline Other & $0.887(0.583-1.351)$ & 0.577 & & \\
\hline Benign & 0.000 & & & \\
\hline Unknown & $1.302(0.715-2.369)$ & 0.388 & & \\
\hline \multicolumn{5}{|c|}{ Pre-operative stage } \\
\hline IA & 1.000 & & 1.000 & \\
\hline IB & 1.870 (1.293-2.705) & 0.001 & $1.744(1.193-2.550)$ & 0.004 \\
\hline \multicolumn{5}{|c|}{ WHO performance status } \\
\hline $0-1$ & 1.000 & & 1.000 & \\
\hline 2 & $1.485(0.960-2.296)$ & 0.076 & $1.281(0.801-2.047)$ & 0.302 \\
\hline $3-4$ & $1.232(0.653-2.326)$ & 0.519 & $0.841(0.416-1.699)$ & 0.629 \\
\hline \multicolumn{5}{|l|}{ Treatment } \\
\hline Surgery & 1.000 & & 1.000 & \\
\hline SABR & 1.309 (0.809-2.118) & 0.272 & $1.271(0.744-2.170)$ & 0.380 \\
\hline CFRT & $2.426(1.472-3.997)$ & 0.001 & $2.133(1.208-3.767)$ & 0.009 \\
\hline
\end{tabular}




\begin{tabular}{|c|c|c|}
\hline & $\operatorname{HR}(95 \% \mathrm{CI})$ & p-value \\
\hline \multicolumn{3}{|c|}{ Surgery } \\
\hline \multicolumn{3}{|c|}{ WHO performance status } \\
\hline $0-1$ & 1.000 & \\
\hline 2 & $1.050(0.536-2.057)$ & 0.887 \\
\hline 3 & $2.911(1.055-8.028)$ & 0.039 \\
\hline \multicolumn{3}{|c|}{ Age group years } \\
\hline$<60$ & 1.000 & \\
\hline $60-69$ & $1.575(0.639-3.885)$ & 0.324 \\
\hline $70-79$ & $2.347(0.987-5.581)$ & 0.054 \\
\hline$\geqslant 80$ & $2.353(0.816-6.785)$ & 0.113 \\
\hline \multicolumn{3}{|c|}{ SABR } \\
\hline \multicolumn{3}{|c|}{ WHO performance status } \\
\hline $0-1$ & 1.000 & \\
\hline 2 & $1.082(0.425-2.756)$ & 0.869 \\
\hline 3 & $0.642(0.201-2.056)$ & 0.456 \\
\hline \multicolumn{3}{|c|}{ Age group years } \\
\hline$<60$ & 1.000 & \\
\hline $60-69$ & $1.608(0.193-13.383)$ & 0.661 \\
\hline $70-79$ & $0.868(0.107-7.037)$ & 0.895 \\
\hline$\geqslant 80$ & $0.655(0.074-5.782)$ & 0.704 \\
\hline \multicolumn{3}{|l|}{ CFRT } \\
\hline \multicolumn{3}{|c|}{ WHO performance status } \\
\hline $0-1$ & 1.000 & \\
\hline 2 & $1.553(0.575-4.199)$ & 0.385 \\
\hline 3 & $0.560(0.144-2.174)$ & 0.402 \\
\hline \multicolumn{3}{|c|}{ Age group years } \\
\hline \multicolumn{3}{|c|}{$<60$} \\
\hline $60-69$ & 1.000 & \\
\hline $70-79$ & $1.249(0.392-3.977)$ & 0.707 \\
\hline$\geqslant 80$ & $1.099(0.357-3.387)$ & 0.870 \\
\hline
\end{tabular}

WHO: World Health Organization; SABR: stereotactic ablative body radiotherapy; CFRT: conventionally fractionated radiotherapy. Univariable outcomes for each treatment and covariable were modelled separately.

survival following SABR was not significantly different to surgery on either univariable or multivariable analysis (multivariable HR 1.271 (95\% CI 0.744-2.170)) (table 3). Excluding patients without histological confirmation prior to surgery resulted in a slight change in the hazard ratio of both SABR and CFRT (HR 1.370 (95\% CI 0.733-2.561) and HR 2.018 (95\% CI 1.004-4.055), respectively) (supplementary table S6). No significant interaction between the impact of age or performance status and treatment modality was demonstrated in the multivariable combined cancer and treatment-related survival Cox model, although the univariable relationship with age and performance status varied between interventions (table 4 and supplementary table S2).

Fine-Gray competing risks analysis confirmed the combined cancer and treatment-related survival analysis findings; SABR outcomes were no different to surgery on multivariable analysis (subdistribution HR 1.030 (95\% CI 0.585-1.814)) (table 5). The cumulative incidence of death due to cancer, treatment or other causes by treatment cohorts is shown in figure 3. The subdistribution HR was 1.149 (95\% CI 0.587-2.250) when patients without histological confirmation were excluded (supplementary table S7).

Second primary cancers were diagnosed in 13 (7.0\%) surgical patients and six (6.1\%) SABR patients; none were seen following CFRT. Diagnosis of recurrence was predominantly clinical in the SABR cohort $(83 \%)$ and histological in the surgical cohort (62\%). Recurrence was predominantly distant in all cohorts (supplementary figure S1). SABR and CFRT had an increased proportion of local recurrences compared with surgery. Further analysis is limited by the differing rates of non-cancer-related death between the cohorts.

The significantly inferior overall survival seen following radiotherapy was further assessed by reversal of the competing risks analysis, with death due to comorbidity considered an event and cancer- or treatment-related deaths competing risks. Despite adjustment in multivariable models, SABR and CFRT 
TABLE 5 Fine-Gray univariable and multivariable competing risks models assessing death due to cancer or treatment with death due to other causes considered a competing risk

\begin{tabular}{ccccc} 
Univariable & & \multicolumn{2}{c}{ Multivariable } \\
\cline { 1 - 1 } $\begin{array}{c}\text { Subdistribution HR } \\
(95 \% \mathrm{CI})\end{array}$ & $\mathrm{p}$-value & & $\begin{array}{c}\text { Subdistribution HR } \\
(95 \% \mathrm{CI})\end{array}$ & $\mathrm{p}$-value
\end{tabular}

\begin{tabular}{|c|c|c|c|c|}
\hline \multicolumn{5}{|c|}{ Age group years } \\
\hline$<60$ & 1.000 & & 1.000 & \\
\hline $60-69$ & $1.799(0.797-4.064)$ & 0.158 & $1.747(0.764-3.990)$ & 0.186 \\
\hline $70-79$ & $2.105(0.956-4.632)$ & 0.064 & $1.914(0.842-4.355)$ & 0.121 \\
\hline$\geqslant 80$ & $1.929(0.824-4.517)$ & 0.130 & $1.590(0.644-3.928)$ & 0.315 \\
\hline \multicolumn{5}{|l|}{ Sex } \\
\hline Female & 1.000 & & 1.000 & \\
\hline Male & $1.267(0.876-1.831)$ & 0.208 & $1.230(0.837-1.805)$ & 0.292 \\
\hline \multicolumn{5}{|c|}{ Pre-operative stage } \\
\hline IA & 1.000 & & 1.000 & \\
\hline $\mathrm{IB}$ & 1.819 (1.259-2.628) & 0.001 & $1.712(1.166-2.515)$ & 0.006 \\
\hline \multicolumn{5}{|c|}{$\begin{array}{l}\text { WHO performance } \\
\text { status }\end{array}$} \\
\hline $0-1$ & 1.000 & & 1.000 & \\
\hline 2 & $1.369(0.887-2.113)$ & 0.156 & $1.256(0.794-1.987)$ & 0.329 \\
\hline $3-4$ & $0.912(0.482-1.728)$ & 0.779 & $0.741(0.342-1.604)$ & 0.446 \\
\hline \multicolumn{5}{|l|}{ Treatment } \\
\hline Surgery & 1.000 & & 1.000 & \\
\hline SABR & $1.000(0.621-1.610)$ & 0.999 & $1.030(0.585-1.814)$ & 0.919 \\
\hline CFRT & $1.848(1.130-3.023)$ & 0.014 & $1.654(0.913-2.996)$ & 0.097 \\
\hline
\end{tabular}

WHO: World Health Organization; SABR: stereotactic ablative body radiotherapy; CFRT: conventionally fractionated radiotherapy.

were associated with significantly greater non-cancer-related mortality (subdistribution HR 2.161 (95\% CI 1.408-3.315); $\mathrm{p}<0.001$ and subdistribution HR 1.868 (95\% CI 1.075-3.243); $\mathrm{p}=0.027$, respectively) (supplementary table S3).

\section{Discussion}

RCTs aiming to compare survival following radical treatment of early-stage lung cancer with surgical resection and SABR have, unfortunately, struggled to recruit. Previous studies have addressed the question of comparative treatment efficacy using observational data. In these studies, however, comparisons are often biased by selection, unobserved confounding and information bias. To the best of our knowledge, this is the only study to deliver an intention-to-treat analysis of patients receiving radical treatment for presumed stage I lung cancer considering separately overall, treatment- and cancer-specific survival outcomes.

The overall survival in this study was comparable to published data [2-6], with the best outcomes observed following surgical resection. SABR and CFRT were associated with inferior overall survival compared with surgery despite adjustment for available baseline characteristics. While CFRT was associated with worse cancer-specific survival and combined cancer and treatment-related survival than surgery, no significant difference in either cancer-specific survival or combined cancer and treatment-related survival was observed between surgery and SABR. The finding that reversal of the competing risks model, considering cancer-related death the competing risk, identified significantly increased non-cancer-related mortality in the radiotherapy treatment arms supports the hypothesis that the overall survival analysis is likely to be biased by unobserved confounding, e.g. due to respiratory function and comorbidity.

Cox modelling of combined cancer and treatment-related survival attempts to assess treatment effect in a theoretical population where death due to other causes is not possible and hence with limited, although not absent, potential for confounding due to comorbidity. Unfortunately, this situation does not reflect the real world and censoring individuals at death due to other causes is open to criticism [30, 31]. It is assumed that those who are censored are represented by the remaining population and unequal censoring between the treatment arms may result in overestimation of the hazard in the more heavily censored (radiotherapy treatment) arms, risking biased estimates of effect [31]. Fine-Gray competing risks analysis provides a means to address this. Notably, however, the risk of cancer recurrence beyond comorbid death 

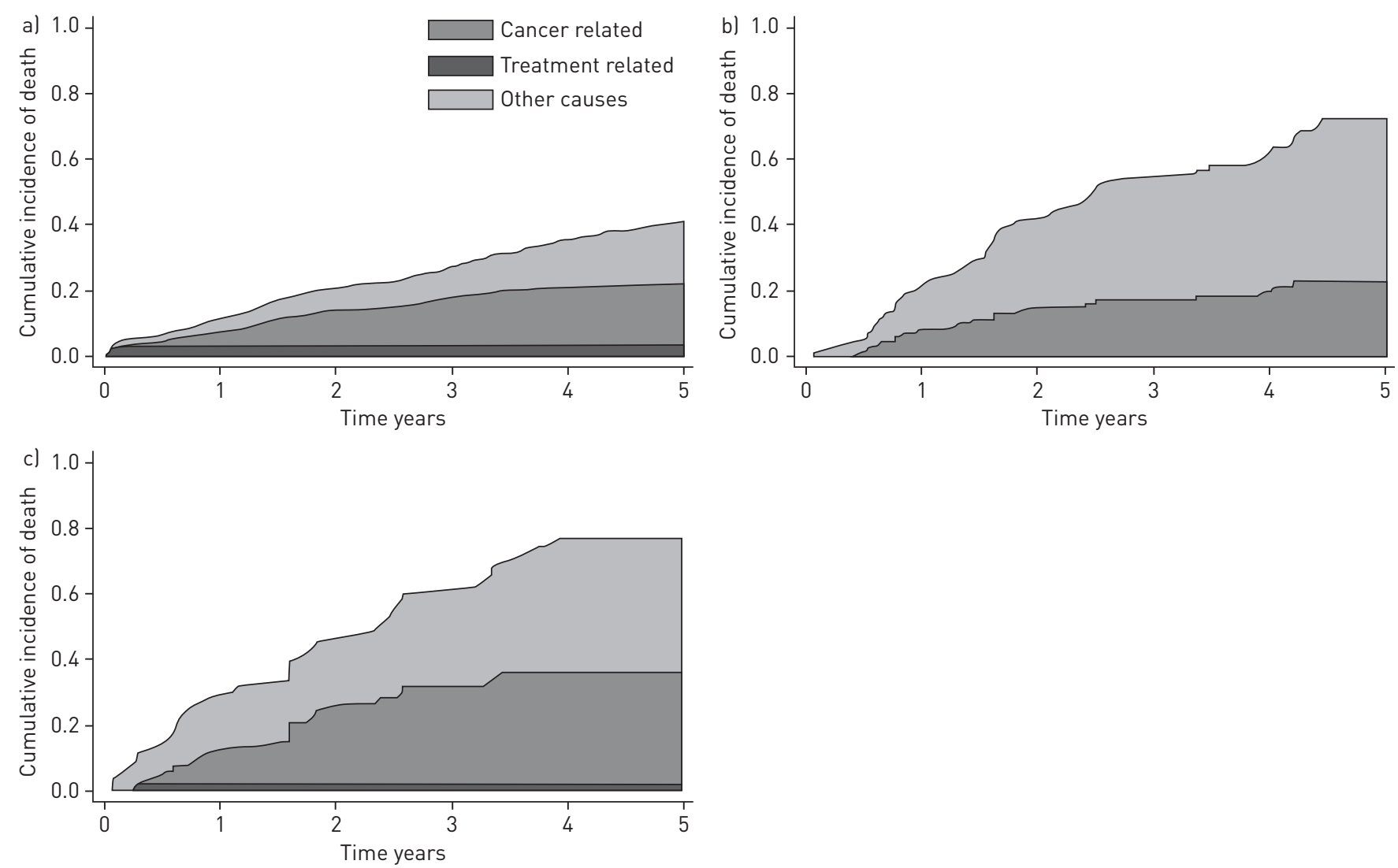

FIGURE 3 Cumulative incidence of death due to cancer, treatment or other causes for a) surgery, b) stereotactic ablative body radiotherapy and c) conventionally fractionated radiotherapy.

remains unobserved. Given the controversy over the optimum methodology, results from both are shown to allow the reader to review the outcomes. Both methods demonstrate no difference in cancer- and treatment-related outcomes between surgery and SABR, while CFRT was associated with inferior combined cancer and treatment-related survival on Cox modelling. These outcomes were unchanged when only individuals with confirmed malignancy prior to treatment were included.

Leeds Cancer Centre has a well-established thoracic surgical service, with six dedicated thoracic surgeons, a dedicated thoracic surgical theatre and high dependency unit, thoracic surgery nurse specialists, and an enhanced recovery programme. The post-operative mortality in this cohort (3.2\%) is in line with national outcomes. The recent Lung Cancer clinical outcomes publication from the UK National Lung Cancer Audit and the Society for Cardiothoracic Surgery in Great Britain and Ireland has reported a 3.8\% 90-day mortality rate after lung cancer resection following 5657 operations in 2014 [8, 32]. In this population, with average operative mortality, no difference is seen in combined cancer and treatment-related survival following SABR. It is notable that the surgically treated population predominantly underwent lobectomy in this cohort. If trials investigating the role of anatomical segmentectomy change current practice, it is not possible to assess the impact this might have on the current results (ClinicalTrials.gov identifier NCT00499330 and [33]).

In order to further assess the factors associated with the outcomes observed, the treatment-specific effects of performance status and age were considered, revealing heterogeneity in treatment effect. Combined cancer and treatment-related survival following surgery appears worse in the older and frailer populations, while these factors had no significant association with survival following radiotherapy. Caution is required in these comparisons; the different sizes of the investigated cohorts makes identification of significant differences more likely in the larger surgical cohort than in the radiotherapy cohorts, although the hazard ratios demonstrated in the radiotherapy cohorts show no suggestion of demonstrating similar effects. Previous studies have identified multiple predictors of surgical mortality and morbidity, including sex, age, comorbidity, baseline pulmonary function and baseline patient reported global health, among others [34, 35]. These findings are also supported by the recent findings of STOKES et al. [36], who demonstrate a significant interaction between age and treatment modality upon the risk of early mortality in a large 
population-based cohort. With the effect of post-operative mortality potentially having a major impact upon the optimal treatment strategy, careful case selection is critical; future research efforts should focus upon helping to predict individual outcomes.

This study has a number of limitations. First, it is a single-centre study, although with a large and diverse population this effect is minimised. Second, despite covering a prolonged time period the cohort remains limited in size. This might limit the potential to identify significant differences in treatment effect, although it is notable that despite this the reverse competing risks analysis identified a significantly higher cumulative incidence of death due to comorbidity in the nonsurgical cohorts. Third, cause of death was identified through retrospective case note review. Primary care records and those from other secondary care centres were not available to support this analysis. It is also acknowledged that this can be challenging prospectively and certainly has limitations retrospectively. All individuals with recurrence were presumed to have died of cancer, while conversely it could be argued that some individuals may have had recurrence that went unrecognised prior to death. The impact of this cannot be assessed, but the two factors may balance each other out. Local recurrence is particularly difficult to identify accurately following SABR. If present, false positives might be worsening the cancer-specific survival and combined cancer and treatment-related survival following SABR, and are therefore unlikely to change the outcome of this study. Fourth, the higher non-cancer-related mortality in the radiotherapy cohorts might be masking increased risk of subsequent cancer recurrence; this remains unobserved. Finally, this study only addresses survival outcomes. The lack of difference in cancer and treatment-related survival outcomes following SABR and surgery demonstrated here make assessment of quality of life outcomes a high priority; this information being of even greater importance to the shared decision-making process where cancer outcomes are similar. Further work is needed, assessing these additional outcomes in larger cohorts and with greater numbers of patients considered eligible for either treatment option.

The data presented in this study demonstrate comparable overall survival [2-6], recurrence rates [5-7] and perioperative mortality to published UK data [8]. Significant differences in this population were observed in non-cancer-related deaths, but no difference in combined cancer and treatment-related survival following SABR and surgery. This finding, however, appears to be masking significant heterogeneity of treatment effect. There is now a pressing need to investigate this further. Randomised trials would be ideal; however, given the challenges of recruitment, high-quality prospective data collection, assessing not only survival but also quality of life, is urgently required to help guide the shared decision-making process.

Acknowledgements: This work uses data provided by patients and collected by the NHS as part of their care and support. We are grateful to Mark Rutherford (University of Leicester, Leicester, UK) for his review and statistical advice.

Conflict of interest: K.L. Spencer has nothing to disclose. M.P.T. Kennedy has nothing to disclose. K.L. Lummis has nothing to disclose. D.A.B. Ellames has nothing to disclose. M. Snee has nothing to disclose. A. Brunelli has nothing to disclose. K. Franks reports personal fees for advisory board work from Pfizer and BMS, personal fees for advisory board work and support for conference attendance from AstraZeneca, and personal fees for educational meetings and support for conference attendance from Boehringer Ingelheim, outside the submitted work. M.E.J. Callister has nothing to disclose.

Support statement: K.L. Spencer was supported by a Wellcome Trust grant and Medical Research Council fellowship (MR/N021339/1) during this project. Funding information for this article has been deposited with the Crossref Funder Registry.

\section{References}

1 Cancer Research UK. Lung cancer incidence statistics. 2015. www.cancerresearchuk.org/health-professional/ cancer-statistics/statistics-by-cancer-type/lung-cancer/incidence Date last accessed: May 16, 2016.

2 Miller DL, Rowland CM, Deschamps C, et al. Surgical treatment of non-small cell lung cancer $1 \mathrm{~cm}$ or less in diameter. Ann Thorac Surg 2002; 73: 1545-1551.

3 Zheng X, Schipper M, Kidwell K, et al. Survival outcome after stereotactic body radiation therapy and surgery for stage I non-small cell lung cancer: a meta-analysis. Int J Radiat Oncol 2014; 90: 603-611.

4 El-Sherif A, Gooding WE, Santos R, et al. Outcomes of sublobar resection versus lobectomy for stage I non-small cell lung cancer: a 13-year analysis. Ann Thorac Surg 2006; 82: 408-416.

5 Ginsberg RJ, Rubinstein LV. Randomized trial of lobectomy versus limited resection for T1 N0 non-small cell lung cancer. Ann Thorac Surg 1995; 60: 615-623.

6 Landreneau RJ, Normolle DP, Christie NA, et al. Recurrence and survival outcomes after anatomic segmentectomy versus lobectomy for clinical stage I non-small-cell lung cancer: a propensity-matched analysis. J Clin Oncol 2014; 32: 2449-2455.

7 Schuchert MJ, Abbas G, Awais O, et al. anatomic segmentectomy for the solitary pulmonary nodule and early-stage lung cancer. Ann Thorac Surg 2012; 93: 1780-1787.

8 Health and Social Care Information Centre. National Lung Cancer Audit 2014: Report for the audit period 2013. 2014. www.hqip.org.uk/resource/national-lung-cancer-audit-report-2014 Date last accessed: January 21, 2019.

9 Powell HA, Tata LJ, Baldwin DR, et al. Early mortality after surgical resection for lung cancer: an analysis of the English National Lung cancer audit. Thorax 2013; 68: 826-834. 
10 Howington JA, Blum MG, Chang AC, et al. Treatment of stage I and II non-small cell lung cancer: diagnosis and management of lung cancer, 3rd edn: American College of Chest Physicians Evidence-Based Clinical Practice Guidelines. Chest 2013; 143: e278S-e313S.

11 Rowell NP, Williams CJ. Radical radiotherapy for stage I/II non-small cell lung cancer in patients not sufficiently fit for or declining surgery (medically inoperable): a systematic review. Thorax 2001; 56: 628-638.

12 Wisnivesky JP, Halm E, Bonomi M, et al. Effectiveness of radiation therapy for elderly patients with unresected stage I and II non-small cell lung cancer. Am J Respir Crit Care Med 2010; 181: 264-269.

13 Gauden SJ, Tripcony L. The curative treatment by radiation therapy alone of stage I non-small cell lung cancer in a geriatric population. Lung Cancer 2001; 32: 71-79.

14 Nyman J, Hallqvist A, Lund J-A, et al. SPACE - a randomized study of SBRT vs conventional fractionated radiotherapy in medically inoperable stage I NSCLC. Radiother Oncol 2016; 121: 1-8.

15 Ball D, Mai T, Vinod S, et al. A randomized trial of SABR vs conventional radiotherapy for inoperable stage I non-small cell lung cancer: TROG 09.02 (CHISEL). J Thorac Oncol 2017; 12: S1853.

16 Palma D, Visser O, Lagerwaard FJ, et al. Impact of introducing stereotactic lung radiotherapy for elderly patients with stage I non-small-cell lung cancer: a population-based time-trend analysis. J Clin Oncol 2010; 28: 5153-5159.

17 Bezjak A, Paulus R, Gaspar LE, et al. Primary study endpoint analysis for NRG Oncology/RTOG 0813 trial of stereotactic body radiation therapy (SBRT) for centrally located non-small cell lung cancer (NSCLC). Int $\mathrm{J}$ Radiat Oncol Biol Phys 2016; 94: 5-6.

18 Lischalk JW, Malik RM, Collins SP, et al. Stereotactic body radiotherapy (SBRT) for high-risk central pulmonary metastases. Radiat Oncol 2016; 11: 528

19 Ricardi U, Badellino S, Filippi AR. Stereotactic radiotherapy for early stage non-small cell lung cancer. Radiat Oncol J 2015; 33: 57-65.

20 Paul S, Lee PC, Mao J, et al. Long term survival with stereotactic ablative radiotherapy (SABR) versus thoracoscopic sublobar lung resection in elderly people: national population based study with propensity matched comparative analysis. BMJ 2016; 354: i3570.

21 Chang JY, Senan S, Paul MA, et al. Stereotactic ablative radiotherapy versus lobectomy for operable stage I non-small-cell lung cancer: a pooled analysis of two randomised trials. Lancet Oncol 2015; 16: 630-637.

22 Subramanian MP, Meyers BF. Surgical resection versus stereotactic body radiation therapy for stage I NSCLC: can randomized trials provide the solution? Cancers 2018; 10: 310.

23 Timmerman R, McGarry R, Yiannoutsos C, et al. Excessive toxicity when treating central tumors in a phase II study of stereotactic body radiation therapy for medically inoperable early-stage lung cancer. J Clin Oncol 2006; 24: 4833-4839.

24 Timmerman R, Paulus R, Galvin J, et al. Stereotactic body radiation therapy for inoperable early stage lung cancer. JAMA 2010; 303: 1070-1076.

25 Westeel V, Barlesi F, Foucher P, et al. 1273O: Results of the phase III IFCT-0302 trial assessing minimal versus CT-scan-based follow-up for completely resected non-small cell lung cancer (NSCLC). Ann Oncol 2017; 28 : Suppl. 5, mdx378.012.

26 Martini N, Melamed MR. Multiple primary lung cancers. J Thorac Cardiovasc Surg 1975; 70: 606-612.

27 Detterbeck FC, Franklin WA, Nicholson AG, et al. The IASLC Lung Cancer Staging Project: background data and proposed criteria to distinguish separate primary lung cancers from metastatic foci in patients with two lung tumors in the forthcoming eighth edition of the TNM Classification for Lung Cancer. J Thorac Oncol 2016; 11: 651-665.

28 Welch HG, Black WC. Are deaths within 1 month of cancer-directed surgery attributed to cancer? J Natl Cancer Inst 2002; 94: 1066-1070.

29 Fine JP, Gray RJ. A proportional hazards model for the subdistribution of a competing risk. J Am Stat Assoc 1999; 94: 496-509.

30 Chappell R. Competing risk analyses: how are they different and why should you care? Clin Cancer Res 2012; 18: 2127-2129.

31 Putter H, Fiocco M, Geskus RB. Tutorial in biostatistics: competing risks and multi-state models. Stat Med 2007; 26: 2389-2430.

32 Royal College of Physicians. Lung cancer clinical outcomes publication 2016 (for the 2014 audit period). 2016. www.rcplondon.ac.uk/projects/outputs/lung-cancer-clinical-outcome-publication-2016-audit-period-2014 Date last accessed: January 21, 2019.

33 Nakamura K, Saji H, Nakajima R, et al. A phase III randomized trial of lobectomy versus limited resection for small-sized peripheral non-small cell lung cancer ( JCOG0802/WJOG4607L). Jpn J Clin Oncol 2010; 40: 271-274.

34 Brunelli A, Salati M, Rocco G, et al. European risk models for morbidity (EuroLung1) and mortality (EuroLung2) to predict outcome following anatomic lung resections: an analysis from the European Society of Thoracic Surgeons database. Eur J Cardiothorac Surg 2017; 51: 490-497.

35 Pompili C, Velikova G, White J, et al. Poor preoperative patient-reported quality of life is associated with complications following pulmonary lobectomy for lung cancer. Eur J Cardiothorac Surg 2017; 51: 526-531.

36 Stokes WA, Bronsert MR, Meguid RA, et al. Post-treatment mortality after surgery and stereotactic body radiotherapy for early-stage non-small-cell lung cancer. J Clin Oncol 2018; 36: 642-651. 